\title{
Drop Head Syndrome as a Rare Complication in Mixed Connective Tissue Disease
}

\author{
Midori Akagi ${ }^{1}$, Masataka Umeda ${ }^{1,2}$, Mikiko Hashisako ${ }^{3}$, Kazusato Hara ${ }^{1}$, Sousuke Tsuji ${ }^{1}$, \\ Yushiro Endo ${ }^{1}$, Ayuko Takatani ${ }^{1}$, Toshimasa Shimizu ${ }^{1}$, Shoichi Fukui ${ }^{1}$, Tomohiro Koga ${ }^{1}$, \\ Shin-ya Kawashiri ${ }^{1}$, Naoki Iwamoto ${ }^{1}$, Takashi Igawa ${ }^{1}$, Kunihiro Ichinose ${ }^{1}$, Mami Tamai ${ }^{1}$, \\ Hideki Nakamura ${ }^{1}$, Tomoki Origuchi ${ }^{4}$, Daisuke Niino ${ }^{5}$ and Atsushi Kawakami ${ }^{1}$
}

\begin{abstract}
:
A 54-year-old woman developed drop head syndrome (DHS), Raynaud's phenomenon and creatine kinase (CK) elevation. She did not meet the international classification criteria of dermatomyositis/polymyositis, as we observed no muscle weakness, grasping pain or electromyography abnormality in her limbs, and antiaminoacyl tRNA synthetase (ARS) antibody was negative. Cervical magnetic resonance imaging and a muscle biopsy of the trapezius muscle revealed myositis findings as the only clinical observations in muscle. These findings, along with her anti-U1-ribonucleoprotein (RNP) antibody positivity and leukopenia, resulted in a diagnosis of mixed connective tissue disease (MCTD). Prednisolone treatment significantly improved her myositis. To our knowledge, this is the first report of DHS as the only muscle complication of MCTD.
\end{abstract}

Key words: mixed connective tissue disease, drop head syndrome, myositis, neck extensor muscles

(Intern Med 59: 729-732, 2020)

(DOI: 10.2169/internalmedicine.3626-19)

\section{Introduction}

Mixed connective tissue disease (MCTD) is an autoimmune disease characterized by overlapping features of more than one autoimmune disease (including systemic lupus erythematosus, polymyositis and scleroderma) with positivity of anti-U1-ribonucleoprotein (RNP) antibody. Myositis is a common complication in patients with MCTD. According to the original case series of MCTD reported by Sharp et al., myositis, defined as symmetrical proximal weakness, electromyographic abnormalities or altered muscle enzymes, was observed in $72 \%$ of patients with MCTD (1). However, anti-RNP antibody-positive myositis of MCTD tends to show milder symptoms, milder histopathological muscle changes and a better response to corticosteroid treatment than polymyositis/dermatomyositis (PM/DM) $(2,3)$.
The frequency of severe myositis in MCTD is rare, but several cases that developed severe and refractory myositis have been reported $(4,5)$. Neck muscles can be affected in patients with MCTD, and myositis in the extensor muscles results in drop head syndrome (DHS). DHS is characterized by kyphotic deformity of the cervico-thoracic spine (6) and is recognized as exceedingly rare complication of inflammatory myopathy. To our knowledge, there is only one report describing DHS in a patient with MCTD who had systemic severe myositis (7).

We herein report a patient with MCTD in whom the only symptom of myositis was DHS and who successfully recovered with oral corticosteroid treatment.

\section{Case Report}

The patient was a 54-year-old Japanese woman had been

\footnotetext{
${ }^{1}$ Department of Immunology and Rheumatology, Nagasaki University Graduate School of Biomedical Sciences, Japan, ${ }^{2}$ Medical Education Development Center, Nagasaki University Hospital, Japan, ${ }^{3}$ Department of Anatomic Pathology, Graduate School of Medicine Sciences, Kyushu University, Japan, ${ }^{4}$ Department of Physical Therapy Sciences, Graduate School of Biomedical Sciences, Nagasaki University, Japan and ${ }^{5}$ Nagasaki Educational and Diagnostic Center of Pathology, Nagasaki University Hospital, Japan

Received: July 9, 2019; Accepted: October 6, 2019; Advance Publication by J-STAGE: November 18, 2019

Correspondence to Dr. Masataka Umeda, masatakau0807@gmail.com
} 


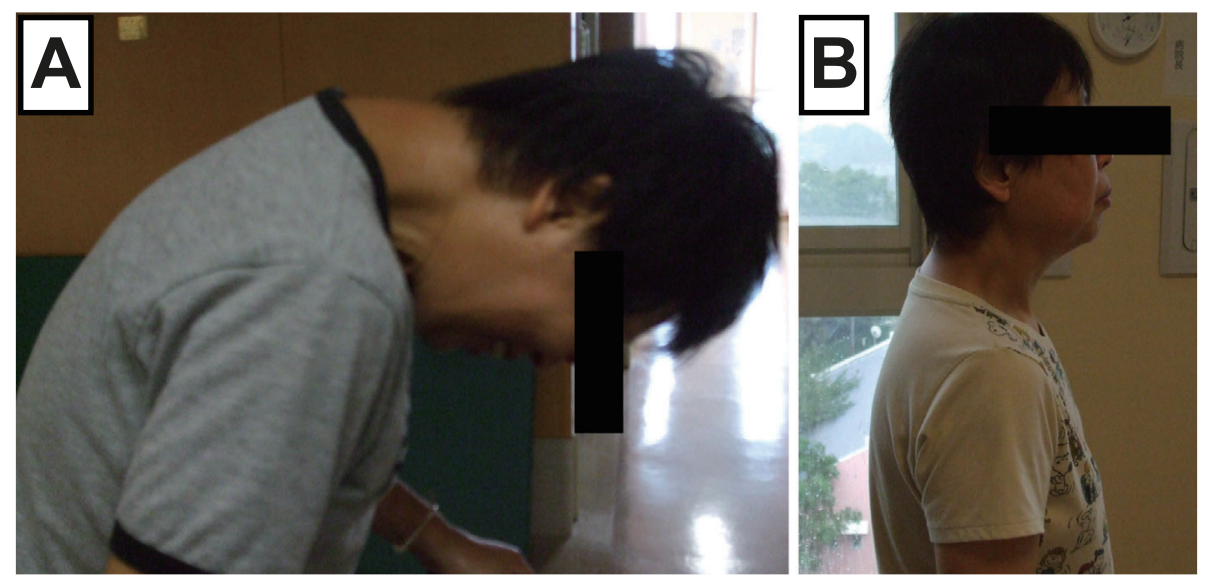

Figure 1. A: The patient showed drop head syndrome (DHS) at an angle of $90^{\circ}$ from the vertical line at admission time. B: Her DHS had completely recovered after four weeks of prednisolone treatment.
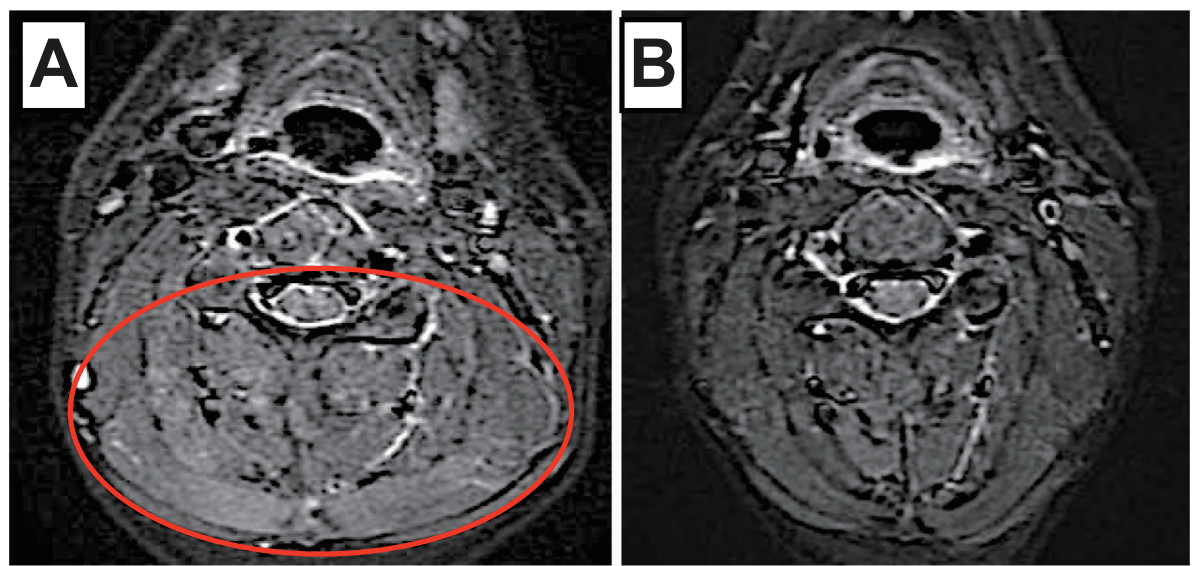

Figure 2. Cervical MRI: short TI inversion recovery (STIR) images. A: High-intensity signals in semispinalis cervicis muscle, semispinalis capitis muscle, splenius cervicis muscle and trapezius muscle (circle). B: The high-intensity signals had decreased after two weeks of prednisolone treatment.

suffering from weakness of the extensor muscles as DHS for 8 months before admission. One month before admission, she had developed worsening of DHS, Raynaud's phenomenon and elevation of CK.

On admission, her body temperature was normal, and a physical examination revealed that the patient had neck extensor muscles weakness (4/5) with DHS (Fig. 1A), but she had neither proximal nor neck flexor muscles weakness or tenderness. Raynaud's phenomenon affected the fingers on both hands without swelling or tenderness of the joints. Normal results were obtained on cardiopulmonary, abdominal and neurologic examinations. She was taking amlodipine, olmesartan medoxomil and magnesium oxide, none of which had been reported to cause myositis.

The initial laboratory studies revealed a white blood cell count of 2,600/L (64.7\% neutrophils, 31.0\% lymphocytes), hemoglobin $10.4 \mathrm{~g} / \mathrm{L}$, platelet count $304,000 / \mu \mathrm{L}$ and erythrocyte sedimentation rate (ESR) $42 \mathrm{~mm} / \mathrm{h}$. Normal results were obtained for aspartate aminotransferase, alanine aminotransferase, electrolytes, C-reactive protein (CRP), C3,
$\mathrm{C} 4 \mathrm{Na}$ urinalysis and thyroid function tests. CK was elevated at 224 IU, but aldolase was normal. Serum antinuclear antibodies (ANA) showed a speckled pattern at a dilution of 1:320, and the anti-U1-RNP antibody levels was $210 \mathrm{U} / \mathrm{mL}$. Anti-aminoacyl tRNA synthetase (ARS) antibodies, anti-MDA5 antibodies, anti-TIF1- $\gamma$ antibodies, antisignal recognition particle (SRP) antibodies, anti-Sm antibodies, anti-dsDNA antibodies, myeloperoxidase antineutrophil cytoplasmic antibody (MPO-ANCA), PR3ANCA, anti-mitochondrial antibody, anti-acetylcholine receptor (AChR) antibodies and anti-Musk antibodies were all negative. An electrocardiogram, chest-abdominal computed tomography, cardiac ultrasonography, limb electromyogram and brain/spinal magnetic resonance imaging (MRI) showed no abnormal findings. However, cervical MRI revealed highintensity signals in the semispinalis cervicis muscles, semispinalis capitis muscles, splenius cervicis muscles and trapezius muscles on short TI inversion recovery (STIR) imaging (Fig. 2A). A biopsy of the trapezius muscle, which also functions as a neck extensor muscle, revealed mild vari- 
ation in the muscle fiber size and degenerated fibers with lymphocytic infiltration (Fig. 3). Slight endomysial fibrosis was also observed.

Based on these results, her clinical findings did not meet the classification criteria of dermatomyositis or polymyositis developed by Bohan and Peter $(8,9)$, the classification criteria of idiopathic inflammatory myopathies (IIM) proposed by the American College of Rheumatology (ACR)/ European League Against Rheumatism (EULAR) 2017 (10) or the diagnostic criteria of PM/DM proposed by Research Committee supported by the Japanese Ministry of Health and Welfare in 2015. However, she did meet the MCTD criteria (Raynaud's phenomenon, positive for anti-RNP antibodies, leukocytopenia, elevated serum levels of muscle enzymes) proposed by the Japanese MCTD Committee supported by the Ministry of Health and Welfare of Japan (11).

The induction of prednisolone at $50 \mathrm{mg}$ /day resulted in the rapid improvement of her symptoms of DHS (Fig. 1B), and the level of CK completely recovered to the normal

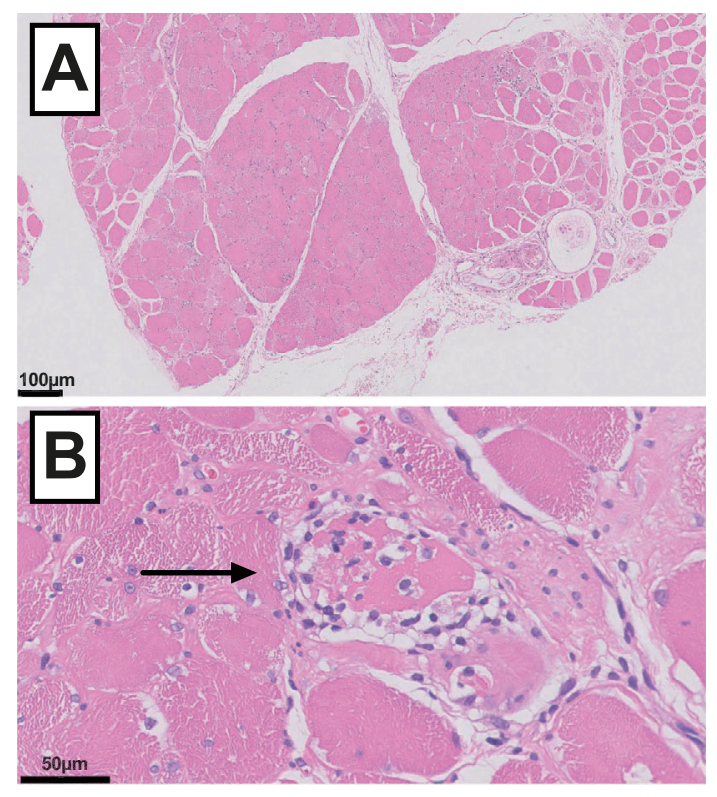

Figure 3. Hematoxylin and Eosin staining of muscle biopsy specimens of the left trapezius muscle where a high intensity on STIR imaging had been noted (A: 10, B: $\times 40$, magnification of the objective lens). The biopsy revealed mild variation in the size of the muscle fibers (A), endomysial infiltration of lymphocytes with degenerated fibers ( $B$, arrow). range. The abnormal high-intensity signals on cervical MRI of the neck extensor muscles also improved (Fig. 2B). Given that her symptom of DHS improved after the initiation of prednisolone therapy, we eventually concluded that the cause of DHS had been myositis of MCTD. In addition, the leukocytopenia and Raynaud's phenomenon were also thought to be complications of MCTD, since both improved following the initiation of treatment. Tapering of the dosage of prednisolone has been successful.

\section{Discussion}

We encountered a rare case of a patient with MCTD who developed myositis in the neck extensor muscle with severe DHS.

DHS is a rare clinical manifestation characterized by weakness of the extensor muscles of the neck, resulting in an inability to raise the head. While DHS is commonly caused by neuromuscular disorders, a broad differential diagnosis is needed (6). According to one systemic literature review, isolated extensor muscle myopathy is the most common cause of DHS $(31.8 \%, 41 / 129)$, while muscle-affecting systemic autoimmune conditions are relatively rare $(7 \%, 9 /$ 129) (12). In IIM, weakness in the neck flexors is reported to be less severe than that in the neck extensors, so DHS is recognized as a rare complication in IIM (10). Focal myositis (FM) which is characterized by localized inflammation of skeletal muscle, also can affect extensor muscles and result in DHS (13). With appropriate intervention of treatment using the optimal diagnostic approach, this condition is reversible; however, the patient's long-term quality of life may be severely affected if the cause cannot be identified promptly (12). The surgical stabilization of the cervical spine may be indicated in refractory cases or cases with an unknown cause $(6,12)$. Inflammatory myopathy arising from autoimmune diseases, such as PM/DM, systemic sclerosis and scleromyositis, are causes of DHS that can be treated with immunosuppression therapies (14). Taken together, these findings suggest that an appropriate diagnosis of autoimmune disease with DHS may result in an optimized therapeutic outcome.

In patients with MCTD, the muscles in the proximal extremities are generally affected by myositis, although the development of cervical myositis is not common (15). The findings of other patients with MCTD who developed symp-

Table. A Summary of the Reports on Patients with Mixed Connective Tissue Disease Developing Symptoms in Neck Muscles.

\begin{tabular}{ccccc}
\hline $\begin{array}{c}\text { Age, } \\
\text { years }\end{array}$ & Sex & Muscle involvement in neck & $\begin{array}{c}\text { Muscle involvement in } \\
\text { other locations }\end{array}$ & References \\
\hline 35 & Woman & Sternocleidomastoid muscle & None & $(16)$ \\
52 & Woman & No detailed description & Lower limbs, dysphagia & $(4)$ \\
57 & Woman & No detailed description & Proximal extremities & $(5)$ \\
41 & Woman & Extensor muscles (drop head syndrome) & Upper limbs & $(7)$ \\
54 & Woman & Extensor muscles (drop head syndrome) & None & Present case \\
\hline
\end{tabular}


toms in the neck muscles are summarized in Table. One case presented with myositis in the sternocleidomastoid muscle as FM, which mimicked jugular thrombophlebitis (16). To our knowledge, the development of DHS in patients with MCTD has only been reported in one case report, in a patient who also had myositis in the upper limbs (7). The present case did not develop systemic myopathic symptoms outside of the neck extensor muscles, nor were any abnormalities noted on a limb electromyogram. These findings suggest that this is the first report of a case in which the only muscle complication of MCTD was DHS with myositis in the extensor muscles.

The present case showed a rapid response to treatment. According to the previous reports, patients who had myositis with positive anti-U1-RNP antibodies had fewer histopathologic changes on a muscle biopsy despite having a similar degree of muscle weakness and $\mathrm{CK}$ levels to patients without anti-U1-RNP antibodies (3). In addition, myositis secondary to MCTD is reported to be more responsive to corticosteroid treatment than that accompanying $\operatorname{IIM}(2,3)$. Of note, in our case, despite the patient's chronic symptoms, the pathology findings in the muscle were relatively mild, which may have been why she showed such a rapid response to treatment.

In conclusion, DHS caused by MCTD is a treatable clinical manifestation once appropriate comprehensively diagnostic approaches are taken. Therefore, patients with DHS should be carefully examined to identify any overlapping features with autoimmune diseases, which may indicate complication with MCTD.

The authors state that they have no Conflict of Interest (COI).

\section{References}

1. Sharp GC, Irvin WS, Tan EM, Gould RG, Holman HR. Mixed connective tissue disease-an apparently distinct rheumatic disease syndrome associated with a specific antibody to an extractable nuclear antigen (ENA). Am J Med 52: 148-159, 1972.

2. Coppo P, Clauvel JP, Bengoufa D, Oksenhendler E, Lacroix C, Lassoued K. Inflammatory myositis associated with anti-U1-small nuclear ribonucleoprotein antibodies: a subset of myositis associated with a favourable outcome. Rheumatology (Oxford, England) 41: 1040-1046, 2002.
3. Lundberg I, Nennesmo I, Hedfors E. A clinical, serological, and histopathological study of myositis patients with and without antiRNP antibodies. Semin Arthritis Rheum 22: 127-138, 1992.

4. S L, Tony K Raghupathy, V S, Malepati B. A rare case of mixed connective tissue disease (MCTD) with intricate features of lupus, polymyositis and rheumatoid arthritis presenting with severe myositis. J Clin Diagn Res 9: OD05-OD07, 2015.

5. Bonin CC, da Silva BS, Mota LM, de Carvalho JF. Severe and refractory myositis in mixed connective tissue disease: a description of a rare case. Lupus 19: 1659-1661, 2010.

6. Martin AR, Reddy R, Fehlings MG. Dropped head syndrome: diagnosis and management. Evid Based Spine Care J 2: 41-47, 2011.

7. Tasca G, Mirabella M, Berrettini A, Monforte M, Tonali PA, Ricci E. Mixed connective tissue disease presenting as a peculiar myositis with poor muscle regeneration. Neurol Sci 32: 171-174, 2011.

8. Bohan A, Peter JB. Polymyositis and dermatomyositis (first of two parts). N Engl J Med 292: 344-347, 1975.

9. Bohan A, Peter JB. Polymyositis and dermatomyositis (second of two parts). N Engl J Med 292: 403-407, 1975.

10. Lundberg IE, Tjarnlund A, Bottai M, et al. International Myositis Classification Criteria Project consortium, The Euromyositis register and The Juvenile Dermatomyositis Cohort Biomarker Study and Repository (JDRG) (UK and Ireland) 2017 European League Against Rheumatism/American College of Rheumatology classification criteria for adult and juvenile idiopathic inflammatory myopathies and their major subgroups. Ann Rheum Dis 76: 19551964, 2017.

11. Kasukawa R, Tojo T, Miyawaki S, et al. Preliminary diagnostic criteria for classifi cation of mixed connective tissue disease. Kasukawa R, Sharp GC, Eds. Excerpta Medica, Amsterdam, 1987: 41-47.

12. Drain JP, Virk SS, Jain N, Yu E. Dropped head syndrome: a systematic review. Clin Spine Surg. Forthcoming.

13. Kastrup A, Gdynia HJ, Nagele T, Riecker A. Dropped-head syndrome due to steroid responsive focal myositis: a case report and review of the literature. J Neurol Sci 267: 162-165, 2008.

14. Fernandez-Serna M, Arboleya L, Alonso S, Queiro R, Alperi M. Dropped head syndrome in a patient with scleromyositis. J Clin Rheumatol 19: 32-34, 2013.

15. Hall S, Hanrahan P. Muscle involvement in mixed connective tissue disease. Rheum Dis Clin North Am 31: 509-517, vii, 2005.

16. Rivest C, Miller FW, Love LA, Turgeon PP, Blier C, Senecal JL. Focal myositis presenting as pseudothrombophlebitis of the neck in a patient with mixed connective tissue disease. Arthritis Rheum 39: 1254-1258, 1996.

The Internal Medicine is an Open Access journal distributed under the Creative Commons Attribution-NonCommercial-NoDerivatives 4.0 International License. To view the details of this license, please visit (https://creativecommons.org/licenses/ by-nc-nd/4.0/).

(C) 2020 The Japanese Society of Internal Medicine Intern Med 59: 729-732, 2020 